\title{
Risk-Based Models of Equipment Repair Management in Power Supply Systems with a Mono Consumer
}

\author{
Yuri A. Sekretarev and Vladimir M. Levin* \\ Novosibirsk State Technical University \\ Novosibirsk, Russian Federation
}

Received 02.02.2021, received in revised form 17.02.2021, accepted 19.02.2021

\begin{abstract}
An adequate description of reliability in decision-making models for managing the restoration of its properties is a complex scientific task. Quite a lot of attention has been paid to the correct solution of this problem in various fields of technology. The article proposes and implements a probabilistic approach to calculating the circuit reliability of the power supply system using an assessment of the actual state of its main elements. The assessment of the state of the equipment of the power supply system is carried out using an integral indicator of the technical condition. This creates the basis for applying a risk-based approach to the management of maintenance and repairs to assess the consequences and feasibility of repair and restoration work of power equipment while minimizing possible damage from power outages to consumers. The article presents two methods for calculating reliability, focused on both recoverable and non-recoverable elements of the power supply system. In addition, a decision-making model for managing repairs of power equipment in power supply systems is proposed. A separate case is considered when the power supply system provides electricity to a mono-consumer, which is typical for oil and gas production. Based on the analysis of scenarios for the implementation of the risk-based approach, the influence of various degrees of severity of the consequences of failure on the assessment of decisions made is studied. The developed models and methods are illustrated by computational examples from the practice of operating electrical equipment in power supply systems with a monoconsumer.
\end{abstract}

Keywords: power supply system, technical condition index, structural reliability of the circuit, probability estimates, risk-based approach, maintenance and repair.

Citation: Sekretarev Yu.A., Levin V.M. Risk-based models of equipment repair management in power supply systems with a mono consumer, J. Sib. Fed. Univ. Eng. \& Technol., 2021, 14(1), 17-32. DOI: 10.17516/1999-494X-0295

(C) Siberian Federal University. All rights reserved

This work is licensed under a Creative Commons Attribution-Non Commercial 4.0 International License (CC BY-NC 4.0).

* Corresponding author E-mail address: levin@corp.nstu.ru 


\title{
Риск-ориентированные модели
}

\section{управления ремонтом оборудования \\ в системах электроснабжения с монопотребителем}

\author{
Ю. А. Секретарев, В. М. Левин \\ Новосибирский государственный технический университет \\ Российская Федерачия, Новосибирск
}

\begin{abstract}
Аннотация. Адекватное описание надежности в моделях принятия решений по управлению восстановлением ее свойств является сложной научной задачей. Корректному решению этой проблемы в различных областях техники уделено достаточно большое внимание. В статье предложен и реализован вероятностный подход к расчету схемной надежности системы электроснабжения с использованием оценки фактического состояния ее основных элементов. Оценка состояния оборудования системы электроснабжения осуществляется с использованием интегрального показателя технического состояния. Это создает основу для применения рискориентированного подхода к управлению техническим обслуживанием и ремонтами для оценки последствий и целесообразности, ремонтно-восстановительных работ энергетического оборудования с минимизацией возможного ущерба от отключений электроэнергии потребителям. В статье представлены два метода расчета надежности, ориентированные как на восстанавливаемые, так и на невосстанавливаемые элементы системы электроснабжения. Кроме того, предложена модель принятия решений по управлению ремонтами энергетического оборудования в системах электроснабжения. Отдельно рассматривается случай, когда система электроснабжения обеспечивает электроэнергией монопотребителя, что характерно для нефтегазодобычи. На основе анализа сценариев реализации риск-ориентированного подхода изучено влияние различной степени тяжести последствий отказа на оценку принимаемых решений. Разработанные модели и методы проиллюстрированы расчетными примерами из практики эксплуатации электрооборудования в системах электроснабжения с монопотребителем.
\end{abstract}

Ключевые слова: система электроснабжения, индекс технического состояния, структурная надежность схемы, вероятностные оценки, риск-ориентированный подход, техническое обслуживание и ремонт.

Цитирование: Секретарев, Ю.А. Риск-ориентированные модели управления ремонтом оборудования в системах электроснабжения с монопотребителем / Ю. А. Секретарев, В. М. Левин // Журн. Сиб. федер. ун-та. Техника и технологии, 2021, 14(1). C. 17-32. DOI: 10.17516/1999-494X-0295

\section{Введение}

Обеспечение надежного и качественного функционирования систем электроснабжения (CЭС) потребителей представляет собой многокритериальную оптимизационную задачу $[1,2]$. Традиционно решения комплексной задачи формируются в трех самодостаточных направлениях. Первое направление связано с выбором оптимального количества функциональных элементов в схеме СЭС, определением необходимых резервов генерирующих или передающих устройств, оптимизацией топологии электрической сети, расчетом и анализом структурной надежности схемы $[3,4,19]$. Второе направление включает выбор качественного состава элементов (оборудования) СЭС, оптимизации их характеристик по критериям надежности и экономичности, а также выбор методов диагностирования и оценки технического состояния электрооборудования $[5,6]$. Третье направление охватывает организацию обеспечения длительной безаварийной 
эксплуатации СЭС, поддержку принятия решений по управлению техническим обслуживанием и ремонтом, оптимизацию управляющих воздействий на оборудование по величине и периодичности $[7,8,20]$. Каждое из перечисленных направлений достаточно формализовано, использует собственный набор критериев и методов анализа. Однако реальная практика анализа и управления надежностью функционирования СЭС расставляет приоритеты для каждого из направлений и указывает на необходимость их комплексного применения.

Применение риск-ориентированного подхода к управлению ремонтами энергооборудования в отечественной и зарубежной практике эксплуатации системы электроснабжения с учетом текущего технического состояния ее элементов и схемной надежности требует регулярного мониторинга последствий отказов оборудования и их влияния на эффективность работы всей системы электроснабжения, включая потребителей [9-11]. Это приводит к неизбежности комбинированного использования локальных критериев и индексов по каждому из указанных направлений в общем алгоритме решения задачи [12-14].

Совершенно очевидно, что значения ущербов у потребителей в технологиях различных производств: химической, нефте- и газодобывающей, пищевой, металлургической, машиностроительной, медицинской, деревообрабатывающей, легкой промышленности и пр., существенно отличаются друг от друга. Это создает практически непреодолимые препятствия для достоверного определения ущербов в тех СЭС, которые обладают всей вышеперечисленной палитрой потребителей. Следует отметить, что нарушение работы СЭС также сопровождается ущербами. Внеплановые и аварийные ремонты энергетического оборудования наносят вполне определенный прямой ущерб, связанный с расходами на производство ремонтных работ и содержанием ремонтного персонала. Изменение режима работы СЭС после отключения отказавшего оборудования вызывает перерасход топлива за счет включения резервных генерирующих источников с худшими экономическими параметрами, что приводит к появлению дополнительного ущерба. Наконец, косвенный ущерб приносит недоиспользование отказавшего энергооборудования и персонала простаивающих установок по их прямому назначению.

Проведенный обзор подчеркивает сложности в оценке индивидуальных ущербов от перерывов электроснабжения потребителей при учете технологических особенностей разных производств и позволяет сформулировать ряд принципиальных выводов, которые могут быть использованы в дальнейших исследованиях:

1. В СЭС, потребители которых представлены широким спектром промышленных производств (полипотребители), достоверная оценка ущербов практически исключена ввиду их многообразия. Еще более нецелесообразным делает их оценку тот факт, что в современных условиях сама СЭС (как производитель и поставщик энергии) и потребитель электроэнергии являются различными субъектами рыночных отношений. В этой связи сам потребитель по мере возможностей и необходимости осуществляет оценку собственного ущерба, а в СЭС ущерб оценивается как сумма отмеченных компонент.

2. Особый случай представляют системы промышленного электроснабжения с монопотребителем (нефте- и газодобывающие комплексы). Здесь система электроснабжения и потребитель представляют единое целое. Более того, режим электроснабжения полностью подчинен технологическим особенностям производства. Выбор стратегии управления такими система- 
ми должен осуществляться на основе ущербов, вызванных отказами как энергетического, так и технологического оборудования всего производственного комплекса.

Именно такие системы электроснабжения представляют особый интерес для исследования, что и определяет содержание данной статьи. В силу случайного характера перечисленных факторов содержание статьи базируется на применении вероятностного подхода к моделированию и проведению необходимых расчетов.

\section{Методология исследования}

Фактическое техническое состояние $i$-й единицы оборудования количественно характеризует интегральный показатель - индекс технического состояния (ИТС) функциональных узлов и единиц электрооборудования (ЭО). Оценка технического состояния единицы ЭО осуществляется сопоставлением фактических значений параметров технического состояния функциональных узлов с их допустимыми (предельно допустимыми) значениями, установленными нормативно-техническими документами, инструкциями заводов-изготовителей, с последующим определением индексов технического состояния функциональных узлов и ЭО в целом. Предложено ИТС единицы ЭО вычислять как средневзвешенное значение ИТС всех функциональных узлов, из которых состоит рассматриваемая единица оборудования, и оценивать в диапазоне от 1,0 (идеальное состояние) до 0 (катастрофическое состояние) с учетом весовых коэффициентов ${ }^{1}$.

Отличие предлагаемого авторами подхода от известных методик отечественных и зарубежных исследователей $[1,4,5,9]$ заключается в полном и объективном учете надежности в СЭС. Как правило, при мониторинге состояния электрооборудования предлагаются упрощенные и неадекватные методики учета надежности. Чаще всего нарушением надежности у потребителя признается только тот случай, когда схема его электроснабжения имеет одну ветвь, которая может выйти из строя за счет отказа любого элемента последовательной цепи: линии электропередачи, трансформатора или выключателя. Если питание потребителя осуществляется по двухцепной линии либо от двух независимых источников, делают вывод, что отказ одной из ветвей не влияет на надежность электроснабжения потребителя. Данное замечание принципиально неверно и противоречит основным законам надежности, согласно которым вывод в аварийный и даже плановый ремонт любого элемента значительно снижает уровень надежности электроснабжения не только в той ветви, в которой это произошло, но и в целом во всей СЭС.

Предлагаемый в статье подход позволяет устранить указанное противоречие за счет простой и эффективной методики расчета схемной надежности электроснабжения и полностью совместить ее с мониторингом и интегральной оценкой технического состояния основного оборудования у монопотребителя. Учет фактического технического состояния единицы ЭО, отличного от идеального, приводит к уменьшению вероятности ее безотказной работы и, соответственно, к увеличению вероятности отказа:

1 Методика оценки технического состояния основного технологического оборудования и линий электропередачи электрических станций и электрических сетей: Утверждена приказом Минэнерго России № 676 от 26.07.2017. [Электронный ресурс]. - Режим доступа: www.ti-ees.ru/fileadmin/f/activity/laws/pr676_260717.pdf. - Заглавие с экрана [Methodology for assessing the technical condition of the main technological equipment and power transmission lines of electric stations and electric networks [Electronic resource] - Access: www.ti-ees.ru/fileadmin/f/activity/laws/ pr676_260717.pdf(In Russia)]. 


$$
Q_{i}=1-\exp (-\omega \cdot t) \cdot J_{i}
$$

где $J_{i}$ - текущее значение индекса технического состояния $i$-й единицы ЭО СЭС. Для определения вероятности безотказной работы необходимо осуществить расчет надежности рассматриваемого участка сети. Вероятность безотказной работы элемента СЭС при условии идеального технического состояния (соответствует $J_{i}=1$ ) рассчитывают следующим образом:

$$
P_{i}=\exp (-\omega \cdot t)
$$

где $\omega$ - значение параметра потока отказа, характеризующее частоту отказов ЭО определенного типа за единицу времени $t$. В зависимости от целей исследования в качестве $t$ может быть рассмотрен либо временной ряд, в течение которого происходит изменение вероятности безотказной работы элемента, либо заданный интервал времени. В статье для демонстрации предлагаемого подхода используется время, равное одному году, что соответствует размерности параметра потока отказов элемента $\left(\omega\right.$, год $\left.{ }^{-1}\right)$. Это упрощает изложение содержания и не искажает сущность предлагаемого подхода.

Если по результатам работы системы мониторинга (диагностирования) $i$-й единицы ЭО определен индекс технического состояния, отличный от идеального $\left(J_{i}<1\right)$, то вероятность безотказной работы будет рассчитана с его учетом как произведение:

$$
P_{\ni i}=P_{i} \cdot J_{i}=\exp \left(\omega_{\ni i}\right) \text { при } t=1 .
$$

Прологарифмировав выражение (3), получим новое значение параметра потока отказов $i$ го элемента СЭС с учетом его фактического технического состояния

$$
\omega_{\ni i}=\ln \left(P_{\ni i}\right) .
$$

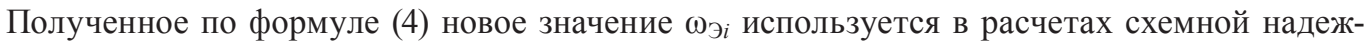
ности СЭС.

Остановимся на методологических подходах к расчету надежности электроснабжения потребителя от СЭС с учетом топологии ее схемы. Элементы схемы могут быть представлены как восстанавливаемые (ремонтопригодные), так и невосстанавливаемые (неремонтопригодные). Восстановление работоспособности неремонтопригодных элементов осуществляется путем замены вышедшего из строя элемента на такой же исправный. Восстановление работоспособности ремонтопригодных элементов производится путем проведения ремонтновосстановительных работ. В связи с этим модели расчета надежности (подходы) для каждого из указанных случаев различны. Ниже излагаются оба подхода к расчету надежности СЭС с учетом ее топологии. Расчет и анализ надежности производится с помощью преобразования исходной схемы электроснабжения в последовательно и параллельно соединенные элементы. Такой метод расчета надежности получил называние метода блок-схем $[15,16,18]$. Согласно указанному методу генерирующие источники объединяют общим узлом генерирующих источников (ШГ), а узел потребителя, для которого определяется уровень надежности электроснабжения, выделяют как ШП. Расчет проводится до тех пор, пока ШГ и ШП не окажутся связанными одним эквивалентным блоком. Показатели надежности эквивалентного блока являются показателями надежности обобщенной схемы электроснабжения, так как он включает в себя все нюансы ее преобразования. 
Первый подход. Расчет надежности схемы электроснабжения, содержащей невосстанавливаемые элементы, осуществляется в вероятностях. При этом вероятность безотказной работы каждого $i$-го элемента рассчитывается как

$$
P_{i}=e^{-\omega}
$$

а вероятность его отказа соответственно

$$
Q_{i}=1-P_{i}
$$

При последовательном соединении элементов схемы СЭС (рис. 1) результирующая для всей группы вероятность безотказной работы определяется по формуле (7), а вероятность отказа - по формуле (8):

$$
\begin{aligned}
& P=\prod_{i=1}^{n} P_{i}, \\
& Q=1-\prod_{i=1}^{n} P_{i} .
\end{aligned}
$$

При параллельном соединении элементов схемы СЭС, которое чаще называется полным резервированием, электроснабжение потребителя будет непрерывным, пока хотя бы один элемент будет находиться в работе (рис. 2).

Результирующая вероятность всей группы элементов может быть определена по формуле (9), а вероятность отказа - по формуле (10):

$$
\begin{aligned}
& P=1-\prod_{i=1}^{n} Q_{i}, \\
& Q=\prod_{i=1}^{n} Q_{i} .
\end{aligned}
$$

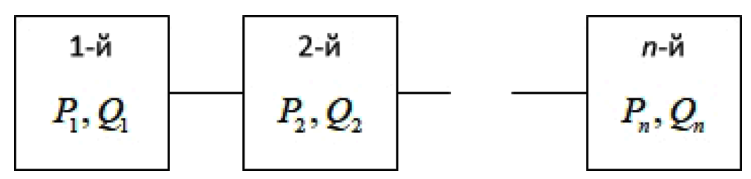

Рис. 1. Схема последовательного соединения элементов СЭС

Fig. 1. Series-connected elements of a PSS

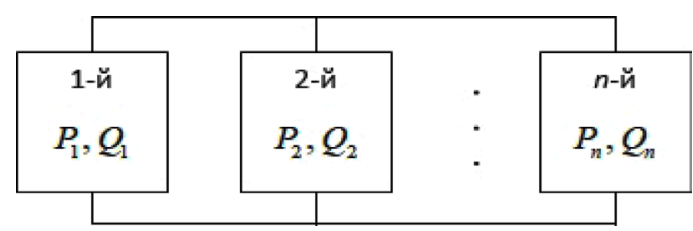

Рис. 2. Схема параллельного соединения элементов СЭС

Fig. 2. Parallel-connected elements of a PSS 
Второй подход. В случае, когда элементы схемы являются восстанавливаемыми (ремонтопригодными), в расчетах помимо параметра потока отказов ( $\omega)$ необходимо определять также и время восстановления $\left(T_{\mathrm{B} i}\right)$, за которое производится восстановление полной работоспособности $i$-го элемента схемы посредством проведения ремонтно-восстановительных работ. При этом для последовательного соединения восстанавливаемых элементов результирующий параметр потока отказов определится как

$$
\omega=\sum_{i=1}^{n} \omega_{i}
$$

Результирующее время восстановления рассчитывается следующим образом:

$$
T_{\mathrm{B}}=\frac{1}{\omega} \sum_{i=1}^{n} \omega_{i} \cdot T_{\mathrm{B} i}
$$

где $\omega_{i}$ имеет размерность (1/год), а $T_{\mathrm{B} i}-$ размерность (ч).

При параллельном соединении двух элементов в группу под параметром потока отказов группы будем понимать следующее:

$$
\omega=\omega_{1} \cdot K_{\mathrm{B} 2}+\omega_{2} \cdot K_{\mathrm{B} 2}(1 / \text { год) }
$$

Здесь $\omega_{1}, \omega_{2}$ - параметры потока отказов, а $K_{\mathrm{B} 1}=\omega_{1} \cdot T_{\mathrm{B} 1}$ и $K_{\mathrm{B} 2}=\omega_{2} \cdot T_{\mathrm{B} 2}-$ коэффициенты вынужденных простоев первого и второго элементов схемы соответственно.

Необходимым условием получения корректного значения результирующего параметра потока отказов $\omega$ по формуле (13) является соблюдение размерности коэффициентов вынужденного простоя $K_{\mathrm{B} 1}$ и $K_{\mathrm{B} 2}$, а именно:

$$
K_{\mathrm{B} 1}=\omega_{1} \cdot \frac{T_{\mathrm{B} 1}}{T_{\mathrm{B} 1}}, K_{\mathrm{B} 2}=\omega_{2} \cdot \frac{T_{\mathrm{B} 2}}{T_{\mathrm{B} 1}}
$$

при обязательном выполнении следующего соотношения: $T_{\mathrm{B} 1}<T_{\mathrm{B} 2}$.

Результирующее время восстановления можно определить по следующей формуле:

$$
T_{\mathrm{B}}=\frac{T_{\mathrm{B} 1} \cdot T_{\mathrm{B} 2}}{T_{\mathrm{B} 1}+T_{\mathrm{B} 2}}
$$

Важным обстоятельством, которое необходимо учитывать при принятии решения о проведении ремонтно-восстановительных работ на элементах оборудования СЭС нефтепромыслов, является выбор критерия (цели) управления. Очевидно, выполнение одного из критериев, сформированных на основе таких показателей надежности, как $Q$ - вероятность отказа энерго-

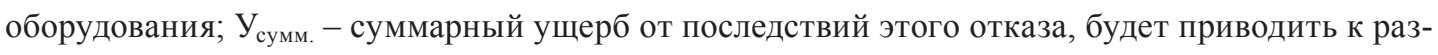
личным результатам:

$$
\begin{aligned}
& Q \rightarrow \min \\
& \text { или } \\
& \mathrm{y}_{\text {сумм. }} \rightarrow \min .
\end{aligned}
$$

Это связано с тем, что большая вероятность отказа единицы ЭО с ухудшенным техническим состоянием не всегда сопутствует большой тяжести последствий (значений потерь добы- 
чи нефти, $\Delta$ П или У). И наоборот, малое значение ущерба от аварийных потерь добычи нефти может наблюдаться при значительной вероятности отказа оборудования. Указанное обстоятельство учитывается при выборе различных стратегий управления ремонтами ЭО. Так, например, критерий (16) направлен на поддержание стратегии ремонтов «по техническому состоянию» ЭО (Condition Based Maintenance), а критерию (17) соответствует стратегия «по оценке прогнозируемых рисков» (Risk Based Maintenance).

Риск отказов ЭО в электрических сетях промышленных предприятий, как правило, связан с нарушениями электроснабжения потребителей, простоями и поломками энергоустановок, угрозами экологии, безопасностью персонала и др. В системах электроснабжения нефтепромыслов основная составляющая риска обусловлена недовыработкой или технологическими потерями добычи нефти $(\Delta \Pi)$. Математическое ожидание ущерба за время восстановления работоспособности аварийно отказавших элементов схемы $T_{\mathrm{B}}$ определяется как вероятностью нарушения электроснабжения технологических потребителей по причине отказа $i$-го элемента сети $\left(Q_{i}\right)$, так и тяжестью возникающих нежелательных последствий (объемом потерь, $\Delta \Pi)$.

Для вычисления вероятности отказа оборудования сети используется, как правило, второй подход, который позволяет определять результирующие показатели надежности восстанавливаемых элементов схемы СЭС, а именно средний параметр потока отказов ( $\omega)$ и среднее время восстановления $\left(T_{\mathrm{B}}\right)$. Расчет значения аварийных потерь добычи нефти выполняется по выражению

$$
\Delta \Pi=\mathrm{P}_{\text {нагр }} \cdot \Delta t \cdot D(\mathrm{~T}),
$$

где $\mathrm{P}_{\text {нагр }}($ кВт) - мощность электрической нагрузки технологических потребителей, потерявших электроснабжение по причине отказа $i$-го элемента сети; $\Delta t=T_{\mathrm{B}}$ (ч) - время перерыва электроснабжения, равное времени восстановления работоспособности $i$-го элемента сети; $D$ (т/кВтч) - удельная производительность нефтедобычи.

Риск аварийного отказа всей схемы электроснабжения, ее отдельного участка или $i$-го элемента определяется как математическое ожидание ущерба от аварийных потерь добычи нефти

$$
\mathrm{M}(\mathrm{Y})=\mathrm{P}_{\text {нагр }} \cdot T_{\mathrm{B}} \cdot D \cdot \mathrm{c} \cdot \omega,
$$

где $c$ - удельная стоимость потерь (руб/т).

\section{Результаты исследований. Анализ и обсуждение}

Представленные в разделе результаты исследований касаются следующих вопросов:

1) анализа и обоснования адекватности разработанных подходов к расчету структурной надежности на примере конкретного участка схемы СЭС нефтепромыслов;

2) оценки влияния значений ИТС ЭО СЭС на надежность электроснабжения потребителя;

3) выбора сценариев для оценки ущербов с учетом дополнительной информации о технологических особенностях нефтедобычи у исследуемого потребителя.

В качестве примера рассмотрен типовой промышленный потребитель, получающий питание от распределительной подстанции 35/6 кВ по двум одноцепным воздушным ли- 
ниям электропередачи напряжением 6 кВ. Схема СЭС исследуемого потребителя показана на рис. 3.

На основании принципиальной схемы составлена блок-схема для расчета структурной надежности СЭС (рис. 4). Исходными данными для расчета являются параметры потока отказов $i$-х элементов схемы $\left(\omega_{i}\right)$ и времена восстановления их работоспособного состояния $\left(T_{\mathrm{B} i}\right)$. Для

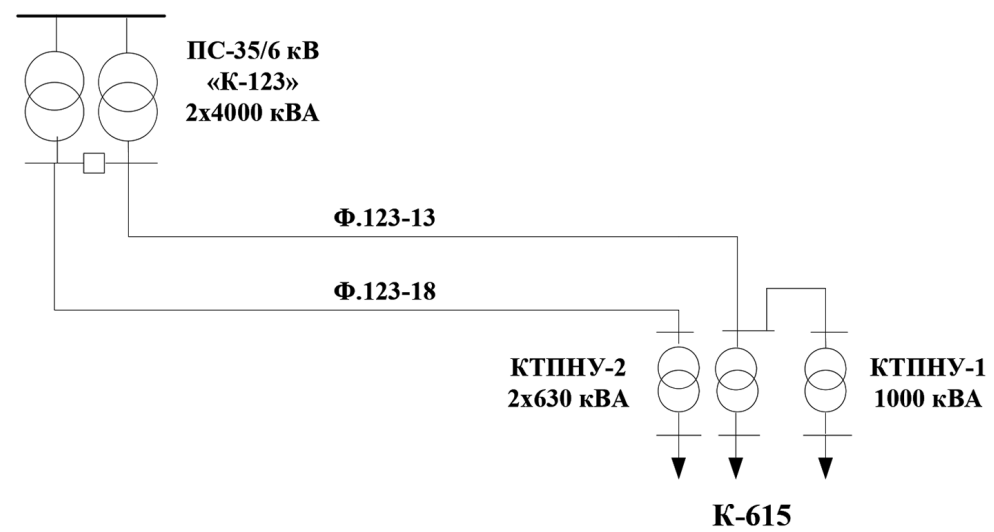

Рис. 3. Схема электроснабжения потребителя

Fig. 3. Circuit diagram of power supply for a consumer

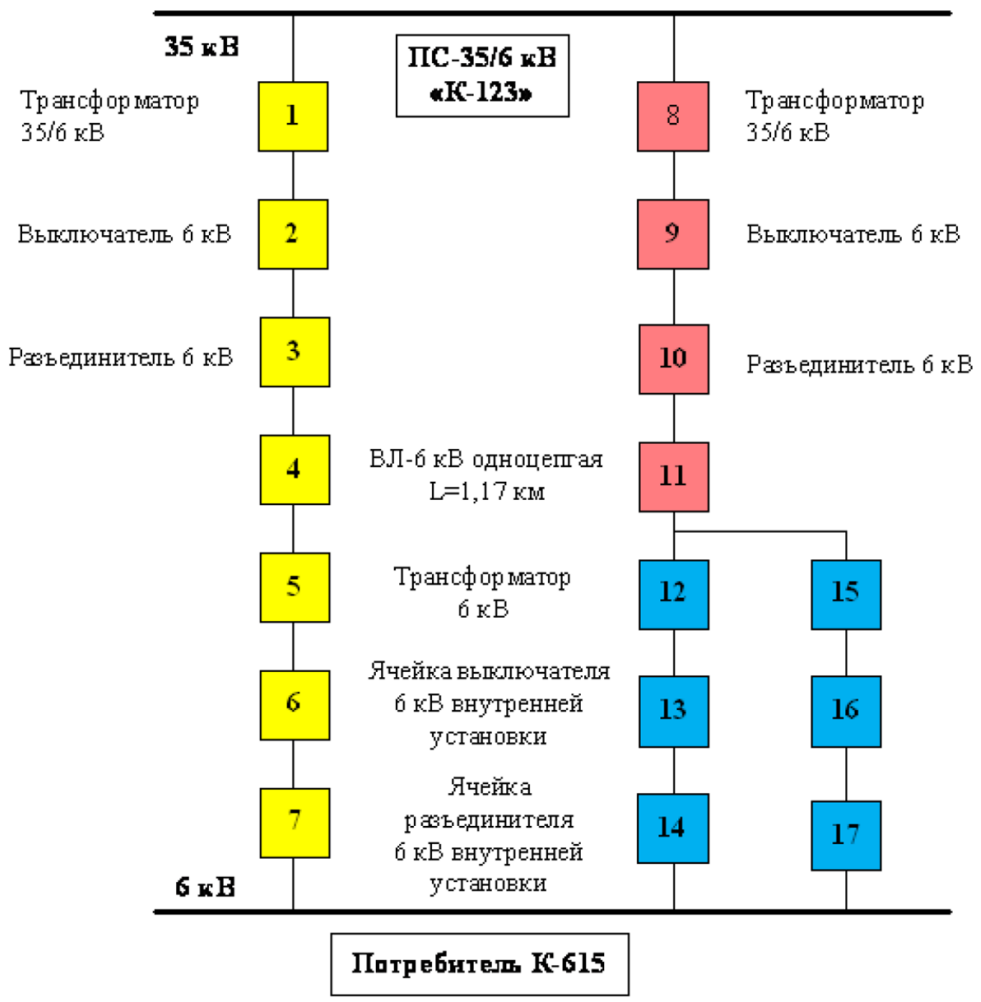

Рис. 4. Блок-схема для расчета надежности электроснабжения

Fig. 4. Block diagram for calculating the power supply reliability 
однозначного понимания, какой из описанных выше подходов применим для адекватной оценки надежности СЭС, проведем расчет надежности указанного объекта по методу блок-схем и проанализируем полученные результаты.

При этом учитывалось следующее:

- элемент А представляет собой последовательно соединенные элементы 1, 2, 3, 4, 5, 6 и 7 (рис. 4);

- элемент В - это последовательно соединенные элементы 8, 9, 10, 11;

- элемент C - это последовательно соединенные элементы $12,13,14$, а элемент $D$ - последовательно соединенные элементы 15, 16, 17;

- элемент Е представляет собой параллельное соединение элементов С и $D$, а элемент $F$ - последовательное соединение элементов В и Е;

- результирующий элемент $R$ - это параллельно соединенные элементы А и $F$. Его показатели надежности полностью характеризуют надежность электроснабжения исследуемого потребителя, относительно которого и велось сворачивание схемы.

В процессе расчетов были получены следующие результаты. Если элементы электрической сети представляются в расчетах надежности как невосстанавливаемые, то вероятность безотказной работы потребителя от источника питания (ПС $35 / 6$ кВ) составит $P^{I}=0,911$, а вероятность отказа $-Q^{I}=1-0,911=0,089$. Если элементы сети представляются в расчетах надежности как восстанавливаемые, то вероятность безотказной работы потребителя от того же источника питания составит $P^{I I}=0,81$, а вероятность отказа будет $Q^{I I}=0,19$. Таким образом, если расчет надежности сети осуществляется с учетом фактора аварийно-восстановительных ремонтных работ на восстанавливаемых элементах схемы, то вероятность отказа увеличивается практически вдвое по сравнению с вариантом невосстанавливаемых элементов. Дадим объяснение отмеченному факту. Во-первых, увеличивается значение параметра потока отказов в схеме с восстанавливаемыми элементами $\left(\omega_{\text {восст. }}=0,21\right)$ по сравнению с невосстанавливаемыми $\left(\omega_{\text {не восст. }}=0,0862\right)$. Это связано с тем, что часть отказов может происходить в момент времени, когда некоторые элементы схемы будут находиться в вынужденном (аварийном) ремонте, что увеличивает суммарный поток отказов. Также увеличивается и время восстановления, поскольку для невосстанавливаемых элементов оно равно времени замены элемента, которое значительно меньше времени восстановления работоспособности элемента (элементов) в результате проведения ремонтных работ. Из этого следует, что расчет надежности схемы электроснабжения необходимо производить с обязательным использованием времени восстановления работоспособности элементов.

Следующий шаг исследования касается расчета значений ИТС и оценки их влияния на надежность полной схемы СЭС. Дополнительно вводили допущение, что на воздушной линии электропередачи (ВЛ) может возникнуть один из двух одиночных дефектов разной степени критичности. В первом случае в качестве такого дефекта принималось нарушение габарита (провис провода), угрожающее схлестыванием фазных проводов $\left(J_{1}\right)$. Во втором случае в качестве дефекта считали наличие на краю просеки угрожающего падением одиночного дерева $\left(J_{2}\right)$. Расчеты ИТС для каждого случая выполняют отдельно по специально разработанной методи- 
ке [17]. По результатам выполненных расчетов ИТС функциональных узлов ВЛ вычисляется обобщенный ИТС для каждого из рассматриваемых видов дефекта:

$$
J_{1}=1 \cdot 0,334+0,37 \cdot 0,666=0,58 ; \quad J_{2}=1 \cdot 0,334+0,73 \cdot 0,666=0,82 .
$$

Далее следует внести оценку ИТС в значение параметра потока отказов ВЛ. Воздушная линия электропередачи 6 кВ в блок-схеме сети (рис. 4) представлена двумя одноцепными ВЛ и фигурирует в виде элементов 4 и 11 . В связи с этим необходимо скорректировать $\omega_{4}$ и $\omega_{11}$, вводя значения ИТС в выражения (3) и (4), (11), (12) и учитывая выражения (13), (14), (15). Результирующие показатели надежности полной схемы электроснабжения в целом с учетом значений $J_{1}$ и $J_{2}$ представлены ниже.

Для первого случая:

$$
\omega_{\text {сумм. }}=1,521 / \text { год. } T_{\text {Всумм. }}=3,41 \text { ч. }
$$

Вероятность безотказной работы полной схемы электроснабжения

$$
\mathrm{P}_{\text {сумм. }}=\exp (-1,52)=0,22 \text {. }
$$

Вероятность отказа схемы электроснабжения будет составлять

$$
Q_{\text {сумм. }}=1-0,22=0,78 .
$$

Для второго случая:

$$
\omega_{\text {сумм. }}=0,6481 / \text { год. } T_{\text {Всумм. }}=3,74 \text { ч. }
$$

Вероятность безотказной работы полной схемы электроснабжения

$$
\mathrm{P}_{\text {сумм. }}=\exp (-0,648)=0,523 \text {. }
$$

Вероятность отказа схемы электроснабжения будет составлять

$$
Q_{\text {сумм. }}=1-0,523=0,477 .
$$

Анализ полученных результатов показывает, что учет ИТС приводит к увеличению вероятности отказа полной схемы электроснабжения в первом случае, от $Q_{\text {сумм. }}=0,19\left(J_{1}=1,0\right.$, состояние идеальное) до $Q_{\text {сумм. }}=0,78$ (ИТС соответствует значению $J_{1}=0,58$ ). Во втором случае отмечается рост вероятности отказа полной схемы электроснабжения от $Q_{\text {сумм. }}=0,19\left(J_{2}=1,0\right)$ до $Q_{\text {сумм. }}=0,477\left(J_{2}=0,82\right)$.

Таким образом, полученные результаты расчетов полностью подтверждают приведенные ранее теоретические положения. В частности, чем хуже техническое состояние элемента (элементов) схемы (ниже значение ИТС), тем выше вероятность отказа исследуемой системы электроснабжения. Для иллюстрации расчета ущербов у потребителя введем в сценарий рассмотренных случаев дополнительную информацию о технологических особенностях нефтедобычи. В первом случае при наличии дефекта, связанного с нарушением габарита (провиса проводов ВЛ), угрожающего их схлестыванием, объектом электроснабжения будет являться технологический потребитель дожимной насосной станции с устройством предварительного сброса пластовой воды (ДНС с УПСВ) мощностью $\mathrm{P}_{\text {нагр }}=1000$ кВт, $\Delta t=1$ ч, $D=0,01$ т/кВтч. 
Во втором случае при наличии дефекта, определенного как угроза падения одиночного дерева на краю просеки, система электроснабжения обеспечивает питание потребителей кустовой площадки нефтяных скважин суммарной мощностью $\mathrm{P}_{\text {нагр }}=1000$ кВт, $D=10$ т/кВтч. При таком высоком дебите получения нефти данный потребитель относится к 1-й категории электроснабжения и оборудован автономным источником питания (дизельной электростанцией, ДЭС) с временем запуска при потере основного и резервного источников питания, равным примерно 1 мин (время автоматической синхронизации и набора нагрузки), т. е. $\Delta t=0,015$ ч. Удельная стоимость потерь добычи нефти принимается единой из расчета 15 тыс. руб. за тонну. В соответствии с выражением (18) определим математическое ожидание ущерба в каждом из рассмотренных случаев.

$$
\mathrm{M}\left(\mathrm{Y}_{1}\right)=1000 \cdot 3,41 \cdot 10 \cdot 15000 \cdot 1,52=744,48 \text { тыс. руб. }
$$

Здесь следует отметить, что при расчете ущерба вместо $\Delta t=1$ ч используется $T_{\text {Всумм. }}=3,41$ ч, полученное при корректном расчете надежности.

$$
\mathrm{M}\left(\mathrm{Y}_{2}\right)=1000 \cdot 0,015 \cdot 0,01 \cdot 15000 \cdot 0,648=1458 \text { тыс. руб. }
$$

В рамках стратегии «по техническому состоянию» приоритетным для выполнения ремонтного воздействия на ВЛ является первый случай с наихудшим ИТС и наибольшей вероятностью отказа $Q_{\text {сумм. }}=0,78$. Однако для него $\mathrm{M}\left(\mathrm{У}_{1}\right)$ составляет 774,48 тыс. руб. В рамках риск-ориентированного подхода приоритет в очередности выполнения ремонта на ВЛ следует отдать второму случаю, который характеризуется меньшей вероятностью отказа $Q_{\text {сумм. }}=0,477$, но более высоким значением математического ожидания ущерба $\mathrm{M}\left(\mathrm{y}_{2}\right)=1458$ тыс. руб. Выбор и принятие решения о выводе элементов СЭС в ремонт по техническому состоянию осуществляется по сценарию, требующему разработки специального алгоритма расчета. Сформулируем исходные данные для примера, схема которого показана на рис. 5. Это условная оперативная схема текущего состояния СЭС с учетом ремонтных режимов, типичная для электроснабже-

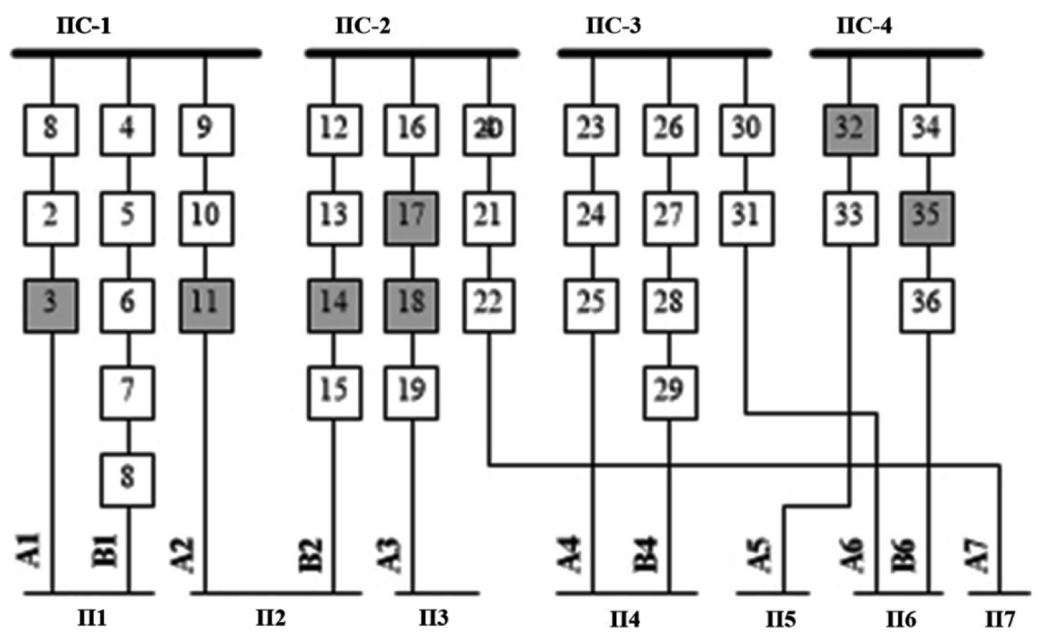

Рис. 5. Оперативная схема системы электроснабжения нефтяного промысла

Fig. 5. Operational diagram of the power supply system of the oil field 
ния нефтяных промыслов. На схеме показаны четыре подстанции (ПС1, ПС2, ПС3, ПС4) и семь потребителей (П1, П1, П2, П3, П4, П5, П6, П7), получающих питание от данных подстанций. Для всех элементов схемы электроснабжения задаются основные показатели надежности (параметр потока отказов и время восстановления), которые могут быть получены либо на основе обработки статистики, либо как нормативно-справочные данные.

Элементы схемы - это трансформаторы, ВЛ, выключатели, разъединители и другое ЭО. Штриховкой на схеме отмечены элементы, на которых диагностический мониторинг выявил определенные дефекты. ИТС этих элементов меньше 1 (техническое состояние ухудшенное).

\section{Алгоритм расчета}

1. Определение элементов со значениями ИТС меньше 1. Такими элементами в примере будут являться $3,11,14,17,18,32,35$.

2. Методом перебора вариантов находят цепочки питания тех потребителей, внутри которых содержатся элементы с ухудшенным значением ИТС. В результате будет получено: Цепочка $\mathrm{A} 1(1,2,3)$ - это потребитель П1. Цепочка A2 $(9,10,11)$ - это потребитель П2. Цепочка В2 $(12,13,14,15)$ - это потребитель П2. Цепочка A3 $(16,17,18,19)$ - это потребитель ПЗ. Цепочка A5 $(\mathbf{3 2}, 33)$ - это потребитель П5. Цепочка А6 $(34, \mathbf{3 5}, 36)$ - это потребитель П6.

3. Осуществляется проверка наличия в схеме дополнительных цепочек питания выбранных потребителей П1, П2, П3, П5, П6 с элементами, обладающими ИТС = 1. В результате проверки такого условия появится еще одна цепочка питания потребителя П1, а именно: Цепочка В1 $(4,5,6,7,8)$ - Потребитель П1.

4. Каждая из идентифицированных выше цепочек состоит из последовательно соединенных элементов, для которых по выражениям (11) и (12) определяют основные результирующие индексы надежности. При этом формируется матрица следующего вида:

$\begin{array}{ll}\text { Потребитель П1 } & \omega_{\mathrm{A} 1} \mathrm{~T}_{\mathrm{BA} 1} \\ \text { Потребитель П2 } & \omega_{\mathrm{B} 1} \mathrm{~T}_{\mathrm{BB} 1} \\ & \omega_{\mathrm{A} 2} \mathrm{~T}_{\mathrm{BA} 2} \\ \text { Потребитель П3 } & \omega_{\mathrm{B} 2} \mathrm{~T}_{\mathrm{BB} 2} \\ \text { Потребитель П5 } & \boldsymbol{\omega}_{\mathrm{A} 5} \mathbf{T}_{\mathbf{B A 5}} \\ \text { Потребитель П6 } & \boldsymbol{\omega}_{\mathbf{A} 6} \mathbf{T}_{\mathbf{B A 6}}\end{array}$

5. Так как потребители П1 и П2 получают питание по двум параллельным цепям (что следует из приведенной матрицы), то для них рассчитывают результирующие индексы надежности по выражениям (11) и (12). При этом окончательный вид матрицы будет следующим:
Потребитель П1 $\boldsymbol{\omega}_{\Pi 1} \mathbf{T}_{\mathbf{B \Pi 1}}$
Потребитель П2 $\boldsymbol{\omega}_{\Pi 2} \mathbf{T}_{\text {вП2 }}$
Потребитель П3 $\boldsymbol{\omega}_{\mathrm{A} 3} \mathbf{T}_{\mathbf{B A} 3}$
Потребитель П5 $\boldsymbol{\omega}_{\mathrm{A} 5} \mathbf{T}_{\mathbf{B A 5}}$
Потребитель П6 $\boldsymbol{\omega}_{\mathrm{A} 6} \mathbf{T}_{\mathbf{B A 6}}$

6. Рассчитывается значение риска по каждому выделенному потребителю. Риск рассчитывается как математическое ожидание ущерба по выражению (18) с учетом технологических особенностей потребителей. В результате рассчитываются риски по каждому потребителю. 


$$
R_{1}=\mathrm{M}\left(\mathrm{y}_{1}\right), R_{2}=\mathrm{M}\left(\mathrm{y}_{2}\right), R_{3}=\mathrm{M}\left(\mathrm{y}_{3}\right), R_{5}=\mathrm{M}\left(\mathrm{y}_{5}\right), R_{6}=\mathrm{M}\left(\mathrm{y}_{6}\right) .
$$

7. Производится ранжирование рисков в порядке их убывания. Предположим, ранжированный ряд будет выглядеть следующим образом:

$$
R_{3}>R_{5}>R_{6}>R_{1}>R_{2}
$$

8. На основании полученного ранжированного ряда формируется приоритезированный список ремонтов элементов СЭС в соответствующей последовательности: 1) элементы 17 и 18; 2) элемент 32; 3) элемент 35; 4) элемент 3; 5) элемент 11 и 14.

9. В соответствии с приоритезированным списком производится планирование графика технического обслуживания и ремонтов ЭО СЭС на календарный год и его оптимизация, включающая ряд дополнительных опций для экономии ресурсов.

\section{Заключение}

1. Предложен вероятностный подход к оценке текущего технического состояния ЭО с расчетом ИТС по результатам его диагностического мониторинга и оценкой структурной надежности схемы СЭС на основе использования двух моделей.

2. Убедительно показано, что при расчете схемной надежности СЭС ее элементы необходимо представлять как восстанавливаемые, поскольку вероятность отказа в этой модели в 2 раза выше, чем упрощенное представление этих элементов в виде невосстанавливаемых. Это объясняется тем, что часть отказов может происходить в момент времени, когда некоторые элементы СЭС уже будут находиться в состоянии вынужденного (аварийного) ремонта, что увеличивает суммарный поток отказов. Также время восстановления работоспособности элемента при проведении ремонтных работ гораздо больше, чем их простая замена после отказа у невосстанавливаемых элементов.

3. Расчет индекса технического состояния ЭО по результатам диагностического мониторинга различных по критичности дефектов и его учет при оценке схемной надежности СЭС значительно увеличивает вероятность нарушения электроснабжения потребителя.

4. В системах электроснабжения с монопотребителем (нефтедобывающий и газовый промыслы), где режимы электроснабжения полностью подчинены технологическим особенностям производства у потребителя, выбор стратегии управления ремонтами должен осуществляться на основе оценки рисков, связанных с отказами как энергетического, так и технологического оборудования всего производственного комплекса.

5. Проведенные исследования и анализ полученных результатов со всей очевидностью показывают, что расчет надежности СЭС необходимо вести с обязательным учетом времени восстановления работоспособности элементов, т. е. использовать подход 2.

6. Чем хуже техническое состояние единицы ЭО, тем выше вероятность отказа системы электроснабжения. В рассмотренном примере уменьшение ИТС с 0,82 до 0,58 приводит к повышению значения $Q$ от 0,477 до 0,78 .

7. Приоритезированная последовательность вывода элементов СЭС в ремонт по техническому состоянию в соответствии с разработанным алгоритмом принятия решений осуществляется на основе минимизации рисков, значения которых рассчитываются как математические

$$
-30-
$$


ожидания ущербов от нарушений электроснабжения потребителей с учетом их технологических особенностей.

\section{Список литературы / References}

[1] Костюков В.Н., Костюков Ал.Н., Костюков Ан.Н. Мониторинг риска эксплуатации оборудования в реальном времени/ Динамика систем, механизмов и машин, 2014, 2, 126-129 [Kostyukov V.N., Kostyukov Al.N., Kostyukov An. N. Monitoring the risk of operating equipment in real time // Dinamika sistem, mekhanizmov i mashin, 2014, 2, 126-129 (in Russian)]

[2] Jiang Y., Zhang Zh., McCalley J.D., Voorhis T.V. Risk-based Maintenance Optimization for Transmission Equipment, Proceedings of the Fifteenth International Conference On Systems Engineering, 2002, 1-7.

[3] Ndawula M.B., Djokic S.Z., Hernando-Gil I. Reliability Enhancement in Power Networks under Uncertainty from Distributed Energy Resources, Energies, 2019, 12(3), 531.

[4] Nordgård D.E., Samdal K. Establishing risk-based maintenance strategies for electricity distribution companies. [Electronic resource] - Access: https://www.sintef.no/globalassets/project/ riskdsam/esrel09-establishing.pdf.

[5] Hashemi-Dezaki H., Askarian-Abyaneh H., Haeri-Khiavi H. Reliability optimization of electrical distribution systems using internal loops to minimize energy not-supplied (ENS), Jornal of Applied Research and Technology, 2015, 13, 416-424.

[6] Горелик А. В., Тарадин Н. А., Веселова А.С., Солдатов Д. В. Оценка качества технической эксплуатации систем железнодорожной автоматики и телемеханики. Автоматика на транспорте, 2017, 3, 319-334 [Gorelik A.V., Taradin N.A., Veselova A.S., Soldatov D. V. Evaluation of the quality of technical operation of railway automation and telemechanics systems. Avtomatika na transporte, 2017, 3, 319-334 (in Russian)]

[7] Чернев М.Ю. Анализ надежности схем электроснабжения на примере Астраханского газоперерабатывающего завода. Промышленная энергетика, 2017, 8, 16-22 [Chernev M. Yu. Analysis of the reliability of power supply schemes on the example of the Astrakhan gas processing plant. Promyshlennaya energetika, 2017, 8, 16-22 (in Russian)]

[8] Singh K. Electricity Network Reliability Optimization. [Electronic resource] - Access: https://hobbydocbox.com/77076786-Radio/Electricity-network-reliability-optimization.html.

[9] Ажмухамедов И.М., Гостюхин Ю.А. Выбор стратегии технического обслуживания и ремонта оборудования сетей связи на предприятиях нефтегазового комплекса. [Электронный ресурс] - Режим доступа: http://www.ivdon.ru/uploads/article/pdf/IVD_142_azhmukhamedov_N. pdf_9487cf0e8c.pdf. - Заглавие с экрана [Azhmukhamedov I.M., Gostyukhin Yu.A. Choosing a strategy for maintenance and repair of communication network equipment at oil and gas complex enterprises [Electronic resource] - Access: http://www.ivdon.ru/uploads/article/pdf/IVD_142_ azhmukhamedov_N.pdf_9487cf0e8c.pdf(In Russia)]

[10] Лесных В.В., Тимофеева Т.Б., Петров В.С. Проблемы оценки экономического ущерба, вызванного перерывами в электроснабжении. Экономика региона, 2017, 13, 3, 847-858. DOI 10.17059/2017-3-17 [Lesnykh V. V., Timofeeva T. B., Petrov V. S. Problems of assessing the economic damage caused by interruptions in power supply. Ekonomika regiona, 2017, 13, 3, 874-858. DOI 10.17059/2017-3-17 (in Russian)] 
[11] Обоскалов В.П. Проблемы расчета структурной надежности систем электроснабжения с использованием вероятностного эквивалентирования. Электричество, 2015, 12, 4-12 [Oboskalov V.P. Problems of calculating the structural reliability of power supply systems using probabilistic equivalence. Elektrichestvo, 2015, 12, 4-12 (in Russian)]

[12] Байдюк М. А., Комарова Г. В. Оценка технического состояния и надежности электрических машин. Известия СПбГЭТУ ЛЭТИ, 2019, 3, 78-84 [Baidyuk M. A., Komarova G. V. Assessment of the technical condition and reliability of electric machines. Izvestiya SPbGETU LETI, 2019, 3, 7884 (in Russian)]

[13] Викторова В.С., Степанянц А.С. Модели и методы расчета надежности технических систем. М.: ЛЕНАНД, 2016. 256 с. [Viktorova V.S., Stepanyants A.S. Models and methods for calculating the reliability of technical systems, Moscow, LENAND, 2016. 256 p. (in Russian)]

[14] Надежность систем энергетики: Проблемы, модели и методы их решения. А. Ф. Дьяков, В.А. Стенников, С. М. Сендеров и др., отв. ред. Н.И. Воропай. Новосибирск: Наука, 2014. 284 c. [Reliability of energy systems: Problems, models and methods of their solution. A. F. Dyakov, V.A. Stennikov, S.M. Senderov et al.; ed. by N.I. Voropai, Novosibirsk, Nauka, 2014. 284 p. (in Russian)]

[15] Гук Ю. Б. Теория надежности в электроэнергетике. М.: Энергоатомиздат, 1990. 208 с. [Guk Yu. B. Theory of reliability in electric power engineering. Moscow, Energoatomizdat, 1990. 208 p. (in Russian)]

[16] Эндрени Дж. Моделирование при расчетах надежности в электроэнергетических системах. М.: Энергоатомиздат, 1983. 336 с. [Andreny G. Modeling in reliability calculations in electric power systems. Moscow, Energoatomizdat, 1983336 p. (in Russian)]

[17] Левин В.М. Управление ремонтами оборудования в системах электроснабжения нефтепромыслов. Электрооборудование: эксплуатация и ремонт, 2019, 6, 41-44 [Levin V.M. Management of equipment repairs in oilfield power supply systems. Elektrooborudovanie: ekspluataciya i remont, 2019, 6, 41-44 (in Russian)]

[18] Allan R.N., Billington R. Reliability Evaluation of Power Systems. Springer Science \& Business Media New York. 1996. 513 p. DOI: 10.1007/978-1-4899-1860-4.

[19] Mehrtash A.; Wang P.; Goel L. Reliability Evaluation of Power Systems Considering Restructuring and Renewable Generators. IEEE Transactions on Power Systems, 2011, 1, 27(1), 243 250. DOI: 10.1109/TPWRS.2011.2161350.

[20] Ndawula M. B., Djokic S.Z., Hernando-Gil, I. Reliability Enhancement in Power Networks under Uncertainty from Distributed Energy Resources. Energies 2019, 12, 3, 531. DOI: 10.3390/ en12030531. 